\title{
New developments in SModelS
}

\section{Gaël Alguero, ${ }^{a, b, *}$ Jan Heisig, ${ }^{c}$ Charanjit K. Khosa, ${ }^{d}$ Sabine Kraml, ${ }^{a, *}$ Suchita Kulkarni, ${ }^{e}$ Andre Lessa, ${ }^{f}$ Philipp Neuhuber, ${ }^{g}$ Humberto Reyes-González, ${ }^{a, d}$ Wolfgang Waltenberger ${ }^{g, h}$ and Alicia Wongel ${ }^{i}$}

${ }^{a}$ Laboratoire de Physique Subatomique et de Cosmologie, Université Grenoble-Alpes, CNRS/IN2P3, 53 Avenue des Martyrs, F-38026 Grenoble, France

${ }^{b}$ LAPTh, Université Savoie Mont Blanc, CNRS, B.P. 110, F-74941 Annecy Cedex, France

${ }^{c}$ Centre for Cosmology, Particle Physics and Phenomenology (CP3), Université catholique de Louvain, Chemin du Cyclotron 2, B-1348 Louvain-la-Neuve, Belgium

${ }^{d}$ Department of Physics, University of Genova, Via Dodecaneso 33, 16146 Genova, Italy

${ }^{e}$ Institute of Physics, NAWI Graz, University of Graz, Universitätsplatz 5, A-8010 Graz, Austria

${ }^{f}$ Centro de Ciências Naturais e Humanas, Universidade Federal do ABC, Santo André, 09210-580 SP, Brazil

${ }^{g}$ Institut für Hochenergiephysik, Österreichische Akademie der Wissenschaften, Nikolsdorfer Gasse 18, A-1050 Wien, Austria

${ }^{h}$ University of Vienna, Faculty of Physics, Boltzmanngasse 5, A-1090 Wien, Austria

${ }^{i}$ DESY, Notkestraße 85, 22607 Hamburg, Germany

E-mail: gael.alguero@lpsc.in2p3.fr, jan.heisig@uclouvain.be, charanjit. kaur@edu. unige.it, sabine.kraml@lpsc.in2p3.fr, suchita.kulkarni@uni-graz.at, andre.lessa@ufabc.edu.br, ph.neuhuber@gmail.com, humbertoalonso.reyesgonzlez@edu.unige.it, wolfgang.waltenberger@oeaw.ac.at, alicia.wongel@desy.de

SModelS is an automatized tool enabling the fast interpretation of simplified model results from the LHC within any model of new physics respecting a $\mathbb{Z}_{2}$ symmetry. In this contribution, we report on two important updates of SModelS during 2020: the extension of the SModelS' database with 13 ATLAS and 10 CMS analyses, including 5 ATLAS and 1 CMS analyses at full Run 2 luminosity, and the ability to use full likelihoods now provided by ATLAS in the form of pyhf JSON files. Moreover, we briefly explain how to use SModelS and give an overview of ongoing developments.

Tools for High Energy Physics and Cosmology - TOOLS2020

2-6 November, 2020

Institut de Physique des 2 Infinis (IP2I), Lyon, France

\footnotetext{
${ }^{*}$ Speaker
} 


\section{Introducing SModelS}

Most experimental searches for new physics at the LHC interpret their data in the context of simplified models. Such simplified models reduce a full theory to only a few new particles with respect to the Standard Model (SM) and assume a simple decay pattern. For example, the supersymmetry (SUSY) motivated search in the $2 \tau+E_{T}^{\text {miss }}$ final state may typically be interpreted in a simplified model that comprises a stau, $\tilde{\tau}_{1}$, and a neutralino, $\tilde{\chi}_{1}^{0}$, as the only new particles and that considers just stau-pair production and a $100 \%$ branching ratio for $\tilde{\tau}_{1} \rightarrow \tau \tilde{\chi}_{1}^{0}$. There is a plethora of ATLAS and CMS analyses from Run 1 and Run 2, looking for new physics in different final states and/or exploiting different analysis techniques, that report their results in the context of such simplified models.

To work out how all these experimental results constrain full models, which may have a large particle content and (much) more complicated decay patterns, is a highly non-trivial task. It may be addressed by reproducing the experimental analyses in Monte Carlo event simulations-this approach, often referred to as "recasting", is however extremely CPU time consuming. Moreover, only a fraction of the experimental searches is implemented in public recasting tools. The alternative is to re-use the published simplified model results - this is the purpose of SModelS [1-6]. This approach requires to compute only cross sections times branching ratios, $\sigma \times \mathrm{BRs}$, for the tested signals. It is often more conservative but much faster than full recasting. Besides speed, the power of SModelS comes from its large database of experimental results, which allows to check limits from nearly 100 ATLAS and CMS searches for SUSY. Note that SModelS can conveniently be used beyond SUSY for any Beyond the Standard Model (BSM) scenario with a $\mathbb{Z}_{2}$-like symmetry, for which the signal acceptance of the SUSY searches apply [1].

In the following, we first present in more detail the working principle of SModelS. This is followed by the new developments published in 2020 (SModelS versions 1.2.3 and 1.2.4), namely a major database update [5] and the pyhf support [6] for usage of full likelihoods provided by ATLAS. We then briefly explain how to use SModelS. We conclude with a short summary, some remarks on the treatment of long-lived particles, and a brief discussion of ongoing developments for the upcoming SModelS version 2.0. An overview of other approaches and recasting tools can be found in [7] and in the review talk by B. Fuks at this workshop.

\subsection{Working principle}

SModelS is based on a general procedure to decompose BSM scenarios featuring a $\mathbb{Z}_{2}$-like symmetry into simplified-model topologies-also referred to as simplified model spectra (SMS). The decomposed topologies are then matched and constrained with experimental results in the SModelS' database (from currently 48 ATLAS and 47 CMS analyses). The working principle is illustrated in Figure 1.

Starting from the BSM particle masses, decay branching ratios and production cross sections provided in the input SLHA or LHE file, SModelS determines the weights in terms of cross sections times branching ratios, $\sigma \times \mathrm{BR}$, of all occurring BSM signals. These signal weights are then confronted with the experimental constraints in the database of LHC results as exemplified in Figure 2. This is easier and much faster than reproducing analyses with Monte Carlo event simulation, and it allows for reinterpreting searches which are not just cut and count, e.g., analyses 


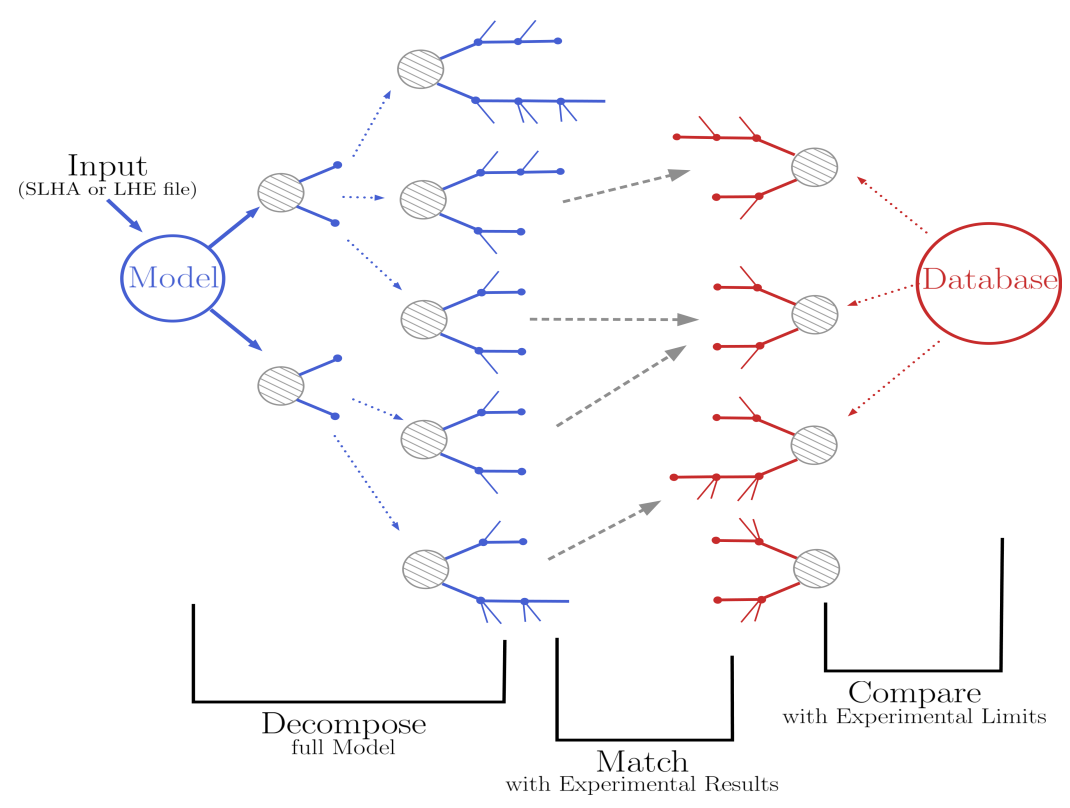

Figure 1: SModelS working principle.

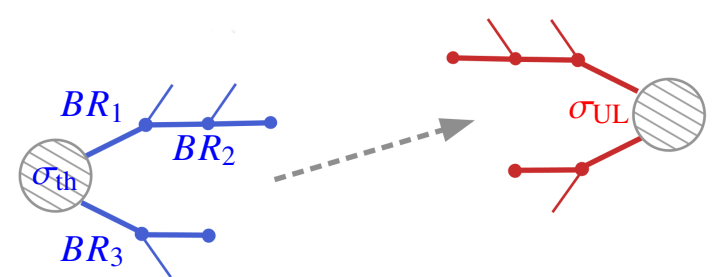

Figure 2: Matching procedure: $\sigma_{\mathrm{UL}}$ sets a limit on $\sigma_{\mathrm{th}} \times B R_{1} \times B R_{2} \times B R_{3}$

which rely on BDT (boosted decision tree) variables. The downside is that the applicability is limited by the simplified model results available in the database (mostly simple, symmetric signal topologies). Moreover, whenever the tested signal splits up into many different channels, as it is often the case in complex models with many new particles, the derived limits tend to be highly conservative.

SModelS is thus particularly useful for evaluating constraints and generally characterizing collider signatures in large scans and model surveys. As mentioned, it can be used for any BSM scenario (beyond the minimal supersymmetric SM, MSSM, and beyond SUSY in general) with a $\mathbb{Z}_{2}$-like symmetry, as long as the simplified model assumptions apply. This means, the kinematic distributions - and thus the signal acceptance - of the tested signal need to be approximately the same as for the (SUSY) scenario assumed for the simplified model in the experimental analysis; ${ }^{1}$ see [1] for a discussion of the simplified model assumptions and associated caveats as well as [8-10] for a quantification of their impact.

\footnotetext{
${ }^{1}$ In particular, the framework does not distinguish between different production modes (e.g. $t$-channel versus $s$-channel) or different spins (see, however, section 5 for upcoming developments).
} 


\subsection{Database structure}

The SModelS database makes use of two types of results: upper limit (UL) results and efficiency map (EM) results. UL maps are 95\% confidence level (CL) upper limits on $\sigma \times$ BR mapped on the parameter space of the given simplified model, typically the BSM masses or slices over the BSM masses. Coming back to the example of the stau-neutralino simplified model (also called TStauStau), the UL map would provide the 95\% CL limit on $\sigma\left(p p \rightarrow \tilde{\tau}_{1} \overline{\tilde{\tau}}_{1}\right) \times\left[\operatorname{BR}\left(\tilde{\tau}_{1} \rightarrow\right.\right.$ $\left.\left.\tau \tilde{\chi}_{1}^{0}\right)\right]^{2}$ in bins of $\left(m_{\tilde{\chi}_{1}^{0}}, m_{\tilde{\tau}_{1}}\right)$. Such UL maps allow us to directly set a limit on the corresponding topology appearing in a full model by interpolating the UL value to the given parameter point. However, their statistical interpretation is limited and only allows for a binary decision - excluded or not - on a topology-per-topology basis. Only if the expected ULs are provided in addition to the observed ones, it becomes possible to select the most sensitive result and/or to estimate an approximate likelihood for the signal strength.

EMs are simulated acceptance times efficiency $(A \times \epsilon)$ values (simply called 'efficiencies' in SModelS) for the various signal regions of an analysis. Like the UL maps above, they are provided as grids in the simplified model parameter space. The big advantage of EMs is that they allow us to combine all contributions to a given signal region from different simplified model topologies. Furthermore, they enable the computation of a proper likelihood, either using a Gaussian approximation for the nuisance parameters (simplified likelihood) [2,3], or using the full statistical model of the analysis [6] (see section 3). They thus enable a richer and more robust statistical inference.

An EM database entry is structured as shown in Figure 3. It has sub-folders for each signal region, here SRhigh and SRlow, which contain the actual EMs, here TStauStau.txt. Moreover, in each signal region sub-folder, there is a dataInfo.txt file with relevant metadata, the number of observed and expected background events and the respective signal upper limits. (We will come back to the globalInfo.txt and . json files in section 3.)

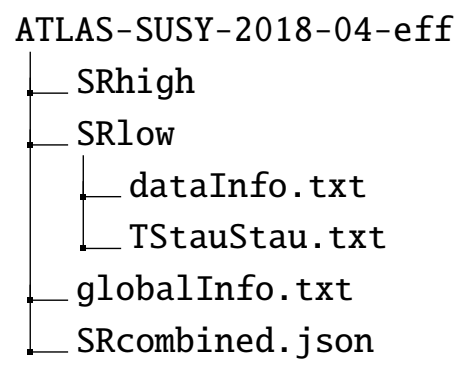

Figure 3: File structure of the ATLAS-SUSY-2018-04 [11] EM database entry

UL database entries are structured analogously, although they do not have specific signal region sub-folders. The database text files are parsed and "pickled", using principal component analysis and Delaunay triangulation, to build a binary database file. The database folder and object structure as well as the binary (pickle) format are explained in detail in the database structure section of the online manual. The whole database may be parsed and pickled locally, or downloaded as a ready-made pickle file. 


\begin{tabular}{lcccl} 
Analysis & $\mathcal{L}\left[\mathbf{f b}^{-1}\right.$ ] & ID & Ref. & Type \\
\hline 0 lept. + jets & 36.1 & SUSY-2016-07 & {$[15]$} & UL \\
0 lept. stop & 36.1 & SUSY-2016-15 & {$[16]$} & UL \\
1 lept. stop & 36.1 & SUSY-2016-16 & {$[17]$} & UL, EM \\
2-3 lept. & 36.1 & SUSY-2016-24 & {$[18]$} & UL, EM \\
photon + jets & 36.1 & SUSY-2016-27 & {$[19]$} & UL, EM \\
0-1 lept. + $b$-jets & 36.1 & SUSY-2016-28 & {$[20]$} & UL \\
EW-ino, Higgs & 36.1 & SUSY-2017-01 & {$[21]$} & UL \\
Higgsino, Z/H & 36.1 & SUSY-2017-02 & {$[22]$} & UL \\
2 OS taus & 139.0 & SUSY-2018-04 & {$[11]$} & UL, EM, JSON \\
3 lept., EW-inos & 139.0 & SUSY-2018-06 & {$[23]$} & UL ${ }^{\star}$ \\
multi- $b$ & 139.0 & SUSY-2018-31 & {$[24]$} & UL, EM, JSON \\
2 OS lept. & 139.0 & SUSY-2018-32 & {$[25]$} & UL \\
1 lept. $+H \rightarrow b \bar{b}$ & 139.0 & SUSY-2019-08 & {$[26]$} & UL, EM, JSON \\
\hline
\end{tabular}

Table 1: Official ATLAS $13 \mathrm{TeV}$ results included in the recent database update. All analyses assume large $E_{T}^{\text {miss }}$ in the final state. The superscript ${ }^{\star}$ denotes UL results which contain expected limits in addition to the observed ones. JSON denotes the availability of full likelihoods, see section 3.

\section{Latest database update}

In the v1.2.3 release [5], the SModelS database was updated with the results from 13 ATLAS and 10 CMS Run 2 analyses. In total, 76 official UL and EM results have been added in v1.2.3 (with 3 more EMs added in v1.2.4). This corresponds to all relevant new ATLAS and CMS results available on HEPData or on the analysis' TWiki page as of end of March 2020. An overview of these new results is given in Tables 1 and 2.

Moreover, 21 "home-grown" EMs were added for two ATLAS and CMS multi-jet $+E_{T}^{\text {miss }}$ searches at $13 \mathrm{TeV}$. These were produced by us using MadAnalysis 5 [12, 13] to improve the coverage of topologies with 2-5 jets shown in Figure 4; these are important for example when gluino-squark associated production is relevant. ${ }^{2}$

Some more comments are in order. Regarding the new ATLAS analyses included in SModelS, half of them have EMs in addition to the UL results, which as mentioned allows for the computation of a likelihood. It is also interesting to observe that five ATLAS analyses are at full Run 2 luminosity and three have full likelihoods available. On the CMS side, more than half of the official results have expected ULs in addition to the observed ones. However, none of them provides EMs. One CMS results is for full Run 2 luminosity.

Figure 5 demonstrates the physics impact of the v1.2.3 database update upon the so-called phenomenological MSSM (pMSSM), i.e. the MSSM with 19 free parameters defined at the weak scale. This makes use of the extensive dataset from the ATLAS pMSSM study [37] available at [38]; concretely we use the "bino LSP" points which are not excluded by the Run 1 searches considered in [37]. The left plot in Figure 5 compares the marginal gluino mass distributions of

\footnotetext{
${ }^{2}$ Note here that home-grown EMs were included in SModelS before, notably for stable heavy charged particles and R-hadrons [3, 14].
} 


\begin{tabular}{lcccc} 
Analysis & $\mathcal{L}\left[\mathbf{f b}^{-1}\right.$ ] & ID & Ref. & Type \\
\hline 0 lept., top tagging & 2.3 & SUS-16-009 & {$[27]$} & $\mathrm{UL}^{\star}$ \\
$\geq 2$ taus & 35.9 & SUS-17-003 & {$[28]$} & UL \\
EW-ino combination & 35.9 & SUS-17-004 & {$[29]$} & UL \\
1 lept. compressed stop & 35.9 & SUS-17-005 & {$[30]$} & $\mathrm{UL}^{\star}$ \\
jets + boosted $H \rightarrow b \bar{b}$ & 35.9 & SUS-17-006 & {$[31]$} & $\mathrm{UL}^{\star}$ \\
2 SFOS lept. & 35.9 & SUS-17-009 & {$[32]$} & $\mathrm{UL}^{\star}$ \\
2 lept. stop & 35.9 & SUS-17-010 & {$[33]$} & $\mathrm{UL}^{\star}$ \\
photon $+(b)$ jets & 35.9 & SUS-18-002 & {$[34]$} & $\mathrm{UL}^{\star}$ \\
0 lept. + jets, MHT & 137.0 & SUS-19-006 & {$[35]$} & $\mathrm{UL}^{\star}$ \\
\hline
\end{tabular}

Table 2: Official CMS $13 \mathrm{TeV}$ results included in the recent database update. All analyses assume large $E_{T}^{\text {miss }}$ in the final state. The superscript ${ }^{\star}$ denotes UL results which contain expected limits in addition to the observed ones.
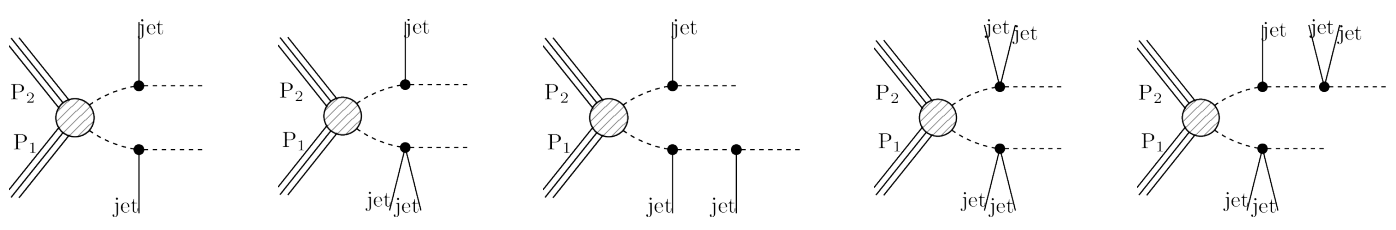

\begin{tabular}{lclll} 
Analysis & $\mathcal{L}\left[\mathbf{f b}^{-1}\right]$ & ID & Ref. & Type \\
\hline 0 lept. + jets & 36.1 & ATLAS-SUSY-2016-07 & {$[15]$} & EM \\
0 lept. + jets & 35.9 & CMS-SUS-16-033 & {$[36]$} & EM \\
\hline
\end{tabular}

Figure 4: Topologies (top row) and analyses (bottom row) for which home-grown EM results were included in the recent database update.

points excluded with v1.2.3 to those excluded with v1.2.2. The plot on the right shows the number of newly excluded points when using only the official ATLAS and CMS results in v1.2.3 (dark red) as compared to using the full v1.2.3 database including the home-grown EMs (orange). The increase in constraining power due to the database update is quite noticeable. In numbers, SModelS v1.2.3 excludes $10 \%$ more points than v1.2.2, 27\% of which are due to the home-grown EMs. Additional plots showing the constraining power can be found in [5].

\section{Interface to pyhf}

As mentioned in section 1.2, EM-type results allow for the construction of a likelihood for the signal strength, and thus for a proper statistical evaluation, including, e.g., the computation of an exclusion limit at a given CL. However, as long as no information on the correlation between signal regions is available, only the most sensitive (a.k.a. "best") signal region should be used for this exercise. This can severely limit the precision of the limit setting, see e.g. contribution 15 of [39]. In turn, as demonstrated in e.g. [40], being able to statistically combine disjoint signal regions instead of using only the best one, can be essential in physics studies. 

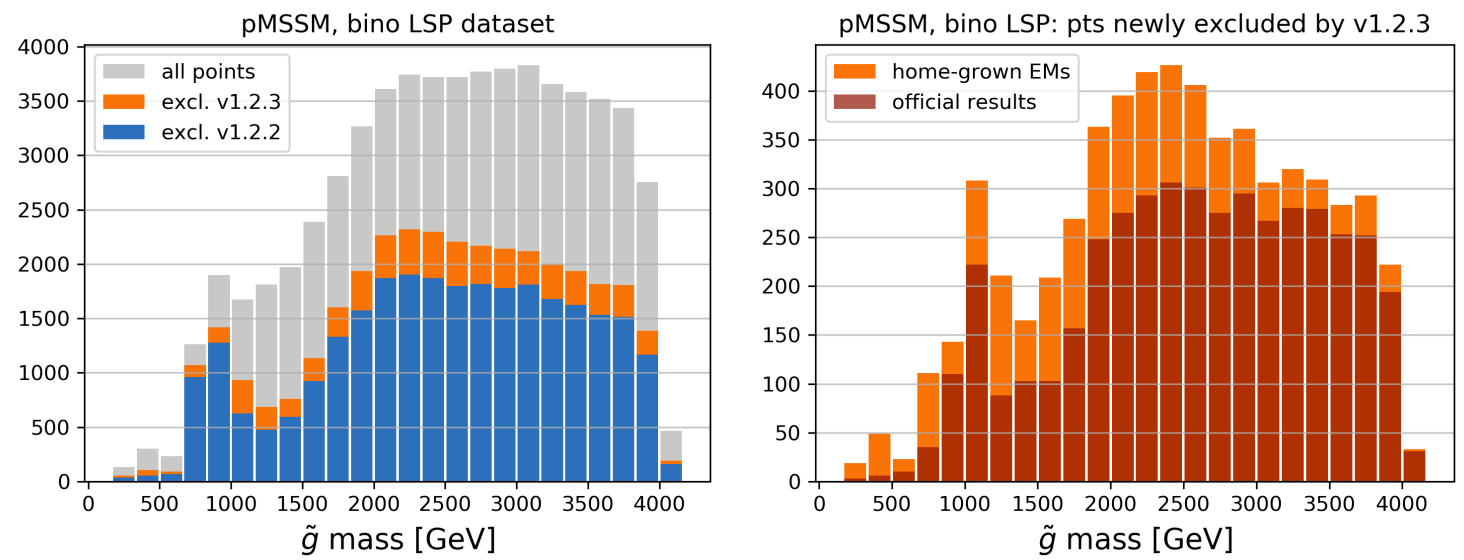

Figure 5: Results for the ATLAS pMSSM points with a bino-like LSP, which were classified as not excluded in [37], as function of the mass of the gluino. Left: In blue the number of points excluded in version 1.2.2, in orange the number of points now excluded in v1.2.3 and in grey the total number of point per bin. Right: the number of newly excluded points when using only the official ATLAS and CMS results in v1.2.3 (dark red) as compared to using the full v1.2.3 database including the home-grown efficiency maps (orange).

The CMS SUSY group has been publishing signal region correlation data in the form of covariance matrices for some of their analyses. This so-called simplified likelihood [41] approach assumes that uncertainties can be well approximated by Gaussians. SModelS can make use of these correlation data since its version 1.2.0 [3].

ATLAS has recently gone a significant step further by publishing full likelihoods using a JSON serialization [42], which provides background estimates, changes under systematic variations, and observed data counts at the same fidelity as used in the experiment. The JSON format describes the HistFactory family of statistical models [43], which is used by the majority of ATLAS searches. The pyhf package [44] is then used to construct statistical models, and perform statistical inference, within a python environment. ${ }^{3}$ The availability of such full likelihoods is a real boon for reinterpretation studies. From SModelSv1.2.4 onwards, we are therefore providing an interface to pyhf, which makes use of the ATLAS JSON files whenever available [6].

As seen in Figure 3, the JSON file describing the likelihood is placed in the folder of the associated database entry; in the example shown, the file is called SRcombined.json. Note that this is a background-only JSON file, i.e. it contains only background numbers with their uncertainties as well as experimentally observed numbers. Upon running SModelS with signal region combination turned on, it is "patched" with the BSM contributions in the various signal regions as determined from the EMs. For this to work correctly, the name(s) of the JSON file(s) to use, as well as the names of the relevant signal regions and their order, are specified in the globalinfo.txt file. (Apart from this, globalinfo.txt contains relevant metadata about the database entry, like the analysis ID, centre-of-mass energy, luminosity, etc.) In our example this is:

datasetOrder: "SRlow", "SRhigh"

jsonfiles: \{"SRcombined.json": ["SRlow", "SRhigh"]\}

\footnotetext{
${ }^{3}$ Note that this fulfills for the first time the Les Houches Recommendations $3 b$ and $3 c$ [45]!
} 

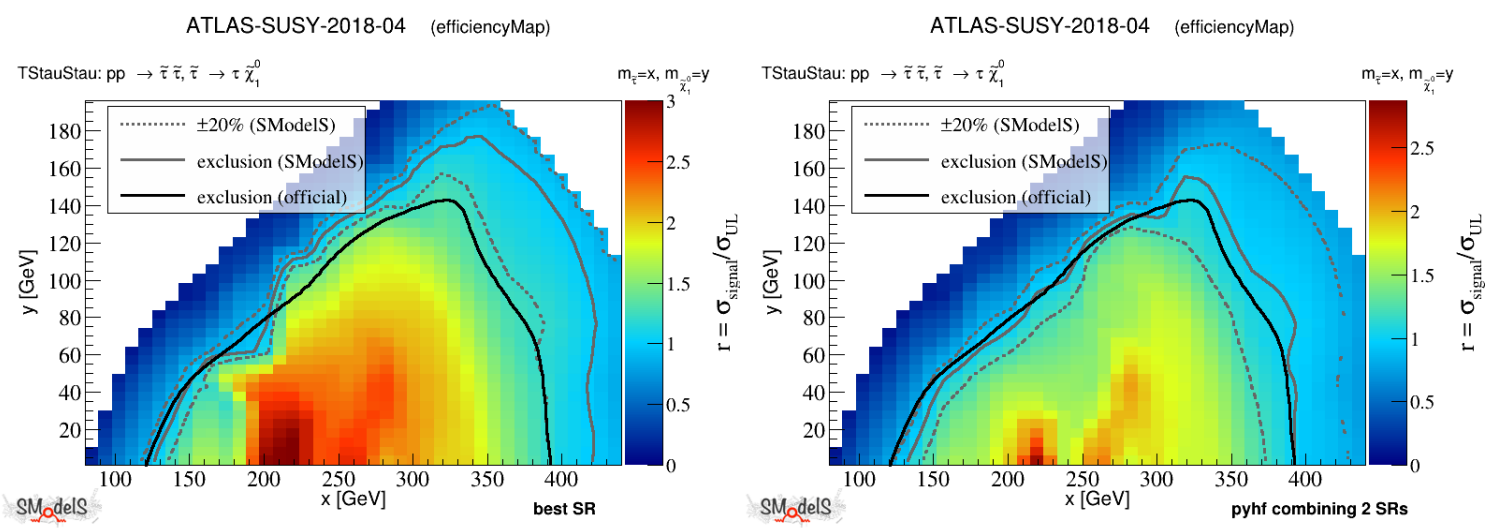

Figure 6: Validation of the TStauStau $\left(p p \rightarrow \tilde{\tau}_{1}^{+} \tilde{\tau}_{1}^{-}, \tilde{\tau}_{1}^{ \pm} \rightarrow \tau \tilde{\chi}_{1}^{0}\right.$ ) result from the ATLAS stau search [11], on the left using the best signal region, on the right using the full likelihood.

pyhf is then used for the statistical evaluation, returning a $\mathrm{CL}_{s}$ that may be varied to infer a cross section upper limit at $95 \%$ exclusion CL.

To demonstrate the physics impact, we compare in Figure 6 the SModelS exclusion (grey line) with the official exclusion (black line) for the ATLAS stau search [11], using the best signal region (left) and using pyhf combination (right). As one can see, the usual procedure, which picks up the most sensitive efficiency map result, over-excludes by about $50 \mathrm{GeV}$ on half the exclusion line. In contrast, a very good agreement with the official ATLAS result is obtained with the full pyhf likelihood. The remaining small difference might be due to the (interpolated) acceptance $\times$ efficiency values from the simplified model EMs not exactly matching the "true" ones of the experimental analysis.

Even though we only show one result here, one can appreciate the gain in accuracy that one can reach with full likelihoods. More examples can be found in [6]. The ATLAS collaboration is at the beginning of a huge effort to provide full statistical models for new analyses. The first analyses published already show how this can help theorists make more trustful reinterpretations. The importance of such likelihood information for, e.g., global fits, has also been emphasised in [7].

\section{Installation and usage}

In this section we provide a short summary of the quick guide for getting started from the tutorial given at this workshop. For more information, the user is kindly referred to the online manual, where a detailed documentation is available.

SModelS is a python library that works with python version 2.6 or later, the default being python3. It is listed in the Python Package Index (PyPI) and can thus be simply installed with pip or pip3 by typing

pip install [--user] smodels

This will install SModelS into the default system or user directory, e.g., $/$.local/lib/. It is therefore recommended only for users who are at ease with working with python libraries. 
Most users may prefer to directly install the package in a local folder using the source file provided on the SModelS releases page on github. To this end, download the source (.tar.gz or .zip) file, extract it at the desired destination and run:

make smodels

in the top-level directory. This will install the required dependencies (using pip install) and compile Pythia [46] and NLL-fast [47]. For the latter two, a C++ and a Fortran compiler are needed. (If the Fortran compiler isn't found, try make $\mathrm{FC}=<$ path-to-gfortran $>$ smodels.) Details on install options and system-specific installations are given in the installation section of the online manual.

The easiest way of using SModelS is via the runSModels. py command-line tool. The basic format is

./runSModelS.py -f <path-to-input-file $>$ [-p parameters.ini]

For the full list of options, type -/runSModelS.py -h. The input file can be an SLHA or an LHE file. If a directory is given, all files in that directory will be processed. Note that input SLHA files need to include masses, decay tables, and LHC production cross sections. (For adding cross sections for MSSM particles in SLHA format, SModelS Tools provides a convenient xsecomputer based on Pythia and NLLfast.) A couple of sample input files for testing are provided in the inputFiles directory.

The basic options and parameters used by runSModels.py are defined in the parameters file. An example including all available parameters together with a short description, is stored in parameters . ini in the top-level directory. Here also the signal region combination can be turned on or off by setting combineSRs to True or False; as signal region combination may take a few seconds per point, False is the default.

Two particularly important settings in the parameters file are the path to the database and the BSM model definition. For the database, one can provide either the path to the local text database, typically

$$
\text { path }=. / \text { smodels-database } /
$$

in which case the database will be parsed and pickled locally, or an URL to the database pickle file. In the latter case, the binary pickle file will be downloaded from the server. This is often faster than pickling locally. Here,

$$
\text { path = official }
$$

provides a short-hand for the official database of your current SModelS version. A list of public database versions is given on the database release page.

The BSM model definition is done in a python file (so-called particle module) that specifies the even and odd particle content of the model. In addition, the quantum numbers of the new particles must be given in a qNumbers dictionary. A couple of pre-defined particle modules are available in smodels/share/models/: mssm.py and nmssm.py for the MSSM and Nextto-MSSM, dgmssm.py for the minimal Dirac gaugino model, and idm.py for the Inert Doublet Model. In the parameters file, the path to the particle module is specified as, e.g., 
model=share. models. idm

If no model is given, the MSSM is taken as the default.

While writing the particle module for a new model is rather straightforward, producing appropriate input SLHA files (including decay tables and cross sections) may be less so. Here the micrOMEGAs-SModelS interface [48], controlled with the switch

\section{\#define SMODELS}

from micrOMEGAs 4.3 onwards, provides a convenient way: for all implemented models, including new ones added by the user, micrOMEGAs automatically produces the needed particle.py and SLHA-style SModelS input files for the parameter point under consideration (decay tables and cross sections are computed at tree level with calcHEP). SModelS is called from micrOMEGAs by the function

smodels(Pcm, nf, csMinFb, fileName, wrt)

where $\mathrm{Pcm}$ is the proton beam energy in $\mathrm{GeV}$ and $\mathrm{nf}$ is the number of parton flavors used to compute the production cross sections of the BSM particles. The result is reported in SLHA format. The details of this functionality are described in [48] and in the manual shipped with micrOMEGAs.

This brings us to the SModelS output. SModelS primarily reports its results in the form of $\boldsymbol{r}$-values, defined as the ratio of the theory prediction over the observed upper limit, for each experimental constraint that is matched in the database. Generally, all points for which at least one $r$-value equals or exceeds unity $\left(r_{\max } \geq 1\right)$ are considered as excluded. For EM-type results, when computeStatistics=True, the likelihood and $\chi^{2}$ are also reported. As an example, Figure 7 shows part of the output obtained for the gluino_squarks.slha sample input file located in inputFiles/slha/.

Moreover, if testCoverage=True, SModelS prints a list of missing topologies: elements which are not tested by any of the experimental results in the database (independent of the element mass). Finally, outside grid elements, which could be tested by one or more experimental result, but are not constrained because the mass array is outside the mass grid, are reported.

Different output formats are available: summary, stdout, log, python, xml, slha. They are chosen and controlled from the parameters.ini file. A detailed explanation of the information contained in each type of output is given in the SModels Output section of the online manual.

Although runSModelS. py provides the main SModelS features with a command line interface, users more familiar with python and the SModelS language may prefer to write their own main program. A simple, commented example code for this purpose is provided as Example.py in the SModelS distribution. The How To's in the online manual provide additional examples for using SModelS and some of the SModelS Tools as a python library.

Last but not least, users can contact the SModelS developers at smodels-users@lists.oeaw.ac.at 


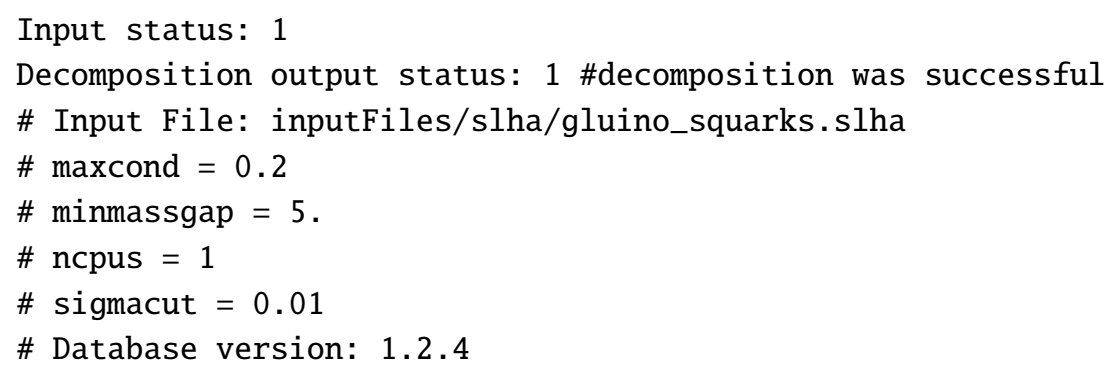

The highest $r$ value is $=7.709501308305477$

Figure 7: Part of the summary output (gluino_squarks.slha.smodels file) obtained when processing ./runSModels.py $-f$ inputFiles/slha/gluino_squarks.slha -p parameters.ini. This is an MSSM scenario with $m_{\tilde{g}}=865 \mathrm{GeV}$ and $m_{\tilde{q}} \approx 990 \mathrm{GeV}$. The results constraining this scenario are reported from highest to lowest $r$-value. In this example, the highest $r$-value comes from the home-grown EMs for the ATLAS-SUSY-2016-07 analysis (see Figure 4), combining the contributions from several signal topologies. The second-highest $r$-value comes from the UL result for the T2 topology from CMS-SUS-19-009 (see Table 2). The "Txnames" nomenclature for the topologies is explained in the SMS dictionary. Note that for the EM result, the $\chi^{2}$ and likelihood are reported in addition to the observed and expected $r$-values. The full output also contains information on unconstrained cross sections (missing topologies and outside grid elements), not displayed here.

for any questions or comments which may arise using the package. New contributors are also always welcome.

\section{Summary and outlook to SModelS 2.0}

We gave a short introduction to constraining new physics with SModelS and presented two important updates of the tool published in 2020: (i) a major database update with results from 13 ATLAS and 10 CMS SUSY searches at $13 \mathrm{TeV}$, and (ii) the interface to pyhf, which enables SModelS to use full likelihoods from ATLAS. The database update significantly increases the constraining power of SModelS and the ability to use full likelihood models leads to a marked gain in accuracy 
(whenever full likelihoods are available).

While the examples discussed in this contribution focus on $E_{T}^{\text {miss }}$ final states, the capabilities of SModelS go beyond $E_{T}^{\text {miss }}$ searches for new physics. Indeed, long-lived particles (LLPs) constrained by searches for heavy stable charged particles (HSCP) or $R$-hadrons, can be treated in SModelS since version $1.2[3,14]$. These searches require the LLP to decay outside the (relevant parts of the) detector, which introduces a dependence on the particle's total decay width. To apply such results to intermediate lifetimes, which lead to a fraction of the decays happening inside the detector, SModelS computes the fractions of prompt decays and decays taking place outside the detector assuming a constant boost factor for the LLP [14]. The impact of the currently implemented HSCP searches has recently been demonstrated for the Dirac gaugino case in [49], where it was shown that their constraining power is at the level of the full recasting approach.

Ongoing developments include a major revision of the decomposition algorithm that enables the support of general LLP signatures. Most significantly, it introduces the particles' decay widths and their quantum numbers as additional parameters of the simplified model topologies. Accordingly, the experimental results in the database can be width dependent. This will enable to incorporate a wide range of exotic signatures, such as searches for disappearing and kinked tracks, displaced jets and leptons as well as delayed jets and photons. This is especially relevant for LLPs with decay lengths of the order of millimeters to a few meters.

The inclusion of the particle quantum numbers in the SMS topologies will provide further opportunities. For instance, it will permit to specify the applicability of certain experimental results to BSM particles with specific spins, allowing to go beyond the assumptions mentioned in footnote 1. Finally, the particle content of full models will be definable through the use of an input SLHA file with QNUMBERS blocks, which will greatly simplify using SModelS for models other than the MSSM. These features will be available with the upcoming SModelS version 2.0.

\section{Acknowledgements}

The work of G.A. and S.K. is supported in part by the IN2P3 project "Théorie - BSMGA". J.H. acknowledges support from the F.R.S.-FNRS, of which he is a postdoctoral researcher. Su.K. acknowledges support from the FWF Elise-Richter fellowship under project number V592-N27.

\section{References}

[1] S. Kraml, S. Kulkarni, U. Laa, A. Lessa, W. Magerl, D. Proschofsky et al., SModelS: a tool for interpreting simplified-model results from the LHC and its application to supersymmetry, Eur.Phys.J. C74 (2014) 2868 [1312.4175].

[2] F. Ambrogi, S. Kraml, S. Kulkarni, U. Laa, A. Lessa, V. Magerl et al., SModelS v1.1 user manual: Improving simplified model constraints with efficiency maps, Comput. Phys. Commun. 227 (2018) 72 [1701.06586].

[3] F. Ambrogi et al., SModelS v1.2: long-lived particles, combination of signal regions, and other novelties, Comput. Phys. Commun. 251 (2020) 106848 [1811.10624]. 
[4] J. Dutta, S. Kraml, A. Lessa and W. Waltenberger, SModelS extension with the CMS supersymmetry search results from Run 2, LHEP 1 (2018) 5 [1803.02204].

[5] C.K. Khosa, S. Kraml, A. Lessa, P. Neuhuber and W. Waltenberger, SModelS database update v1.2.3, LHEP 158 (2020) 2020 [2005.00555].

[6] G. Alguero, S. Kraml and W. Waltenberger, A SModelS interface for pyhf likelihoods, 2009.01809.

[7] LHC Reinterpretation Forum, Reinterpretation of LHC Results for New Physics: Status and Recommendations after Run 2, SciPost Phys. 9 (2020) 22 [2003.07868].

[8] L. Edelhäuser, J. Heisig, M. Krämer, L. Oymanns and J. Sonneveld, Constraining supersymmetry at the LHC with simplified models for squark production, JHEP 12 (2014) $022[1410.0965]$.

[9] L. Edelhäuser, M. Krämer and J. Sonneveld, Simplified models for same-spin new physics scenarios, JHEP 04 (2015) 146 [1501.03942].

[10] S. Kraml, U. Laa, L. Panizzi and H. Prager, Scalar versus fermionic top partner interpretations of $t \bar{t}+E_{T}^{\text {miss }}$ searches at the LHC, JHEP 11 (2016) 107 [1607.02050].

[11] ATLAS collaboration, Search for direct stau production in events with two hadronic $\tau$-leptons in $\sqrt{s}=13 \mathrm{TeV}$ p p collisions with the ATLAS detector, Phys. Rev. D 101 (2020) 032009 [1911.06660].

[12] B. Dumont, B. Fuks, S. Kraml, S. Bein, G. Chalons, E. Conte et al., Toward a public analysis database for LHC new physics searches using MADANALYSIS 5, Eur. Phys. J. C 75 (2015) 56 [1407. 3278].

[13] E. Conte and B. Fuks, Confronting new physics theories to LHC data with MADANALYSIS 5, Int. J. Mod. Phys. A 33 (2018) 1830027 [1808.00480].

[14] J. Heisig, S. Kraml and A. Lessa, Constraining new physics with searches for long-lived particles: Implementation into SModelS, Phys. Lett. B788 (2019) 87 [1808. 05229].

[15] ATLAS collaboration, Search for squarks and gluinos in final states with jets and missing transverse momentum using $36 \mathrm{fb}^{-1}$ of $\sqrt{\mathrm{s}}=13 \mathrm{TeV}$ pp collision data with the ATLAS detector, Phys. Rev. D97 (2018) 112001 [1712. 02332].

[16] ATLAS collaboration, Search for a scalar partner of the top quark in the jets plus missing transverse momentum final state at $\sqrt{s}=13 \mathrm{TeV}$ with the ATLAS detector, JHEP 12 (2017) 085 [1709.04183].

[17] ATLAS collaboration, Search for top-squark pair production in final states with one lepton, jets, and missing transverse momentum using $36 \mathrm{fb}^{-1}$ of $\sqrt{s}=13 \mathrm{TeV}$ pp collision data with the ATLAS detector, JHEP 06 (2018) 108 [1711.11520]. 
[18] ATLAS collaboration, Search for electroweak production of supersymmetric particles in final states with two or three leptons at $\sqrt{s}=13 \mathrm{TeV}$ with the ATLAS detector, Eur. Phys. J. C78 (2018) 995 [1803.02762].

[19] ATLAS collaboration, Search for photonic signatures of gauge-mediated supersymmetry in 13 TeV pp collisions with the ATLAS detector, Phys. Rev. D97 (2018) 092006 [1802. 03158].

[20] ATLAS collaboration, Search for supersymmetry in events with b-tagged jets and missing transverse momentum in p collisions at $\sqrt{s}=13 \mathrm{TeV}$ with the ATLAS detector, JHEP 11 (2017) 195 [1708.09266].

[21] ATLAS collaboration, Search for chargino and neutralino production in final states with a Higgs boson and missing transverse momentum at $\sqrt{s}=13 \mathrm{TeV}$ with the ATLAS detector, Phys. Rev. D100 (2019) 012006 [1812.09432].

[22] ATLAS collaboration, Search for pair production of higgsinos in final states with at least three b-tagged jets in $\sqrt{s}=13 \mathrm{TeV}$ p p collisions using the ATLAS detector, Phys. Rev. D98 (2018) 092002 [1806.04030].

[23] ATLAS collaboration, Search for chargino-neutralino production with mass splittings near the electroweak scale in three-lepton final states in $\sqrt{s}=13 \mathrm{TeV}$ p p collisions with the ATLAS detector, Phys. Rev.D 101 (2020) 072001 [1912.08479].

[24] ATLAS collaboration, Search for bottom-squark pair production with the ATLAS detector in final states containing Higgs bosons, b-jets and missing transverse momentum, JHEP 12 (2019) 060 [1908.03122].

[25] ATLAS collaboration, Search for electroweak production of charginos and sleptons decaying into final states with two leptons and missing transverse momentum in $\sqrt{s}=13 \mathrm{TeV} p \mathrm{p}$ collisions using the ATLAS detector, Eur. Phys. J. C80 (2020) 123 [1908.08215].

[26] ATLAS collaboration, Search for direct production of electroweakinos in final states with one lepton, missing transverse momentum and a Higgs boson decaying into two b-jets in ( $p p$ ) collisions at $\sqrt{s}=13 \mathrm{TeV}$ with the ATLAS detector, Eur. Phys. J. C 80 (2020) 691 [1909.09226].

[27] CMS collaboration, Search for supersymmetry in the all-hadronic final state using top quark tagging in pp collisions at $\sqrt{s}=13$ TeV, Phys. Rev. D96 (2017) 012004 [1701.01954].

[28] CMS collaboration, Search for supersymmetry in events with a $\tau$ lepton pair and missing transverse momentum in proton-proton collisions at $\sqrt{s}=13 \mathrm{TeV}, \mathrm{JHEP} 11$ (2018) 151 [1807.02048].

[29] CMS collaboration, Combined search for electroweak production of charginos and neutralinos in proton-proton collisions at $\sqrt{s}=13$ TeV, JHEP 03 (2018) 160 [1801.03957].

[30] CMS collaboration, Search for top squarks decaying via four-body or chargino-mediated modes in single-lepton final states in proton-proton collisions at $\sqrt{s}=13 \mathrm{TeV}$, JHEP 09 (2018) 065 [1805.05784]. 
[31] CMS collaboration, Search for Physics Beyond the Standard Model in Events with High-Momentum Higgs Bosons and Missing Transverse Momentum in Proton-Proton Collisions at 13 TeV, Phys. Rev. Lett. 120 (2018) 241801 [1712.08501].

[32] CMS collaboration, Search for supersymmetric partners of electrons and muons in proton-proton collisions at $\sqrt{s}=13 \mathrm{TeV}$, Phys. Lett. B790 (2019) 140 [1806. 05264].

[33] CMS collaboration, Searches for pair production of charginos and top squarks in final states with two oppositely charged leptons in proton-proton collisions at $\sqrt{s}=13 \mathrm{TeV}, \mathrm{JHEP} 11$ (2018) 079 [1807.07799].

[34] CMS collaboration, Search for supersymmetry in events with a photon, jets, b -jets, and missing transverse momentum in proton-proton collisions at $13 \mathrm{TeV}$, Eur. Phys. J. C79 (2019) 444 [1901.06726].

[35] CMS collaboration, Search for supersymmetry in proton-proton collisions at $13 \mathrm{TeV}$ in final states with jets and missing transverse momentum, JHEP 10 (2019) 244 [1908.04722].

[36] CMS collaboration, Search for supersymmetry in multijet events with missing transverse momentum in proton-proton collisions at 13 TeV, Phys. Rev. D96 (2017) 032003 [1704.07781].

[37] ATLAS collaboration, Summary of the ATLAS experiment's sensitivity to supersymmetry after LHC Run 1 - interpreted in the phenomenological MSSM, JHEP 10 (2015) 134 [1508.06608].

[38] http://hepdata.cedar.ac.uk/view/ins1389857.

[39] G. Brooijmans et al., Les Houches 2019 Physics at TeV Colliders: New Physics Working Group Report, in 11th Les Houches Workshop on Physics at TeV Colliders: PhysTeV Les Houches, 2, 2020 [2002.12220].

[40] P. Asadi, M.R. Buckley, A. DiFranzo, A. Monteux and D. Shih, Digging Deeper for New Physics in the LHC Data, JHEP 11 (2017) 194 [1707. 05783].

[41] CMS collaboration, Simplified likelihood for the re-interpretation of public CMS results, Tech. Rep. CMS-NOTE-2017-001, CERN, Geneva (Jan, 2017).

[42] ATLAS collaboration, Reproducing searches for new physics with the ATLAS experiment through publication of full statistical likelihoods, Tech. Rep. ATL-PHYS-PUB-2019-029, CERN, Geneva (Aug, 2019).

[43] ROOT collaboration, K. Cranmer, G. Lewis, L. Moneta, A. Shibata and W. Verkerke, "HistFactory: A tool for creating statistical models for use with RooFit and RooStats." https: //cds. cern. ch/record/1456844, 6, 2012.

[44] L. Heinrich, M. Feickert and G. Stark, scikit-hep/pyhf: v0.5.2, Sept., 2020, https://doi.org/10.5281/zenodo.4018115. 
[45] S. Kraml et al., Searches for New Physics: Les Houches Recommendations for the Presentation of LHC Results, Eur. Phys. J. C 72 (2012) 1976 [1203.2489].

[46] T. Sjöstrand, S. Ask, J.R. Christiansen, R. Corke, N. Desai, P. Ilten et al., An Introduction to PYTHIA 8.2, Comput. Phys. Commun. 191 (2015) 159 [1410.3012].

[47] W. Beenakker, C. Borschensky, M. Krämer, A. Kulesza, E. Laenen, S. Marzani et al., $N L O+N L L$ squark and gluino production cross-sections with threshold-improved parton distributions, Eur. Phys. J. C 76 (2016) 53 [1510.00375].

[48] D. Barducci, G. Belanger, J. Bernon, F. Boudjema, J. Da Silva, S. Kraml et al., Collider limits on new physics within micrOMEGAs_4.3, Comput. Phys. Commun. 222 (2018) 327 [1606.03834].

[49] M.D. Goodsell, S. Kraml, H. Reyes-González and S.L. Williamson, Constraining Electroweakinos in the Minimal Dirac Gaugino Model, SciPost Phys. 9 (2020) 047 [2007.08498]. 